\title{
Development of Novel Method for the Detection of Microcystin Using Chemiluminescence Immunochromatography
}

\author{
Dongjin Pyo* and Jisun Yoo \\ Department of Chemistry, Kangwon National University, Chunchon 200-701, Korea. *E-mail: pyod@kangwon.ac.kr \\ Received July 7, 2010, Accepted November 4, 2010
}

\begin{abstract}
A new chemiluminescence immunochromatographic analysis system with high sensitivity and high reproducibility was developed for the determination of microcystins (MCs) in water. Horse radish peroxidase (HRP) labeled microcystin monoclonal antibody was used for the sensitive chemiluminescence detection. The chemiluminescence immunochromatographic analysis system was composed of microcystin LR (MCLR)-monoclonal antibody (mAb)-Horse Radish Peroxidase (HRP) conjugate, MCLR-BSA conjugate, luminol, hydrogen peroxide mixture solution, an immunochromatographic assay strip and luminometer. To detect the concentration of microcystins in water, we utilized one spot analysis of the strip instead of flow type analysis. We could detect the microcystins in water at a concentration as low as $9.45 \mathrm{pg} / \mathrm{mL}$ with the chemiluminescence (CL) detection.
\end{abstract}

Key Words: Microcystin, Monoclonal antibodies, Chemiluminescence, Immunochromatography

\section{Introduction}

In many countries, the presence of cyanobacteria in freshwater bodies used for both drinking water and recreational purpose is under increasing public health attention. The main issue about cyanobacteria in freshwater is their ability to produce potent cyanotoxins, presenting waterborne hazards to human and animals. Since drinking water is essential in the human and animal health, it is very important to develop fast, reliable, and accurate analytical methods to detect cyanobacterial toxin, microcystins in water.

Immunoassays, with high selectivity and affinity of antibody molecules to their corresponding antigens, have widely been exploited for analytical purposes in the fields of clinical diagnoses. ${ }^{1-4}$ Some immunoassays for microcystin have been reported such as microchip based assay, ${ }^{5}$ enzyme phosphataseinhibition assay, ${ }^{6}$ lateral flow immunoassay, ${ }^{7}$ enzyme-linked immunosorbent assay (ELISA), ${ }^{8}$ and chemical methods have been employed for the determination of microcystin, such as high performance liquid chromatography (HPLC) $)^{9}$ and tandem mass spectrometry (LC/MS/MS). ${ }^{10}$ While these methods have their own advantages, they are still inconvenient since they require time consuming sample pretreatment procedure, expensive equipments and qualified experts.

Chemiluminescence (CL) is the light emission derived from a chemical reaction in which chemically excited molecules decay to the electronic ground state and emit photons. CL is generated when a chemical reaction gives an electronically excited product, which emits radiation to convert back to the resting state. The CL method is a powerful analytical tool because of high sensitivity, wide linear range and simple instrumentation without a light source. The primary reason for high sensitivity and selectivity is that only a few compounds have CL characteristics. In the case of luminol oxidation catalyzed by peroxidase, the decay of aminophthalate dianion provides light emission $(\lambda=$ $425 \mathrm{~nm}) .{ }^{11,12}$ In aqueous solutions the light observed ranges between blue violet and blue-green. CL detection techniques use horseradish peroxidase (HRP) or alkaline phosphatase (ALP) as labeled to the antibody or antigen molecules. CL can be an essential tool in clinical, medical, pharmaceutical and environmental analysis. ${ }^{11}$

Luminol (5-amino-2,3-dihydro-1,4-phthalazine-dione) is a cyclic acyl-hydrazide, and shows the typical reactivity of this class of compounds (Figure 1). ${ }^{2}$ White et al. observed that the fluorescence spectrum of an intermediate molecule in the luminol oxidation process named 3-aminophthalate in an electronically excited state (3-APA*) perfectly matched the chemiluminescence spectrum of luminol, thus they concluded that this excited intermediate could be considered the light emitting species upon deexcitation to the ground state (3-APA). ${ }^{13-15}$ This was confirmed in 1965 by Gundermann. ${ }^{16}$ In dipolar aprotic solvents such as dimethylsulfoxide (DMSO) containing $\mathrm{O}_{2}$, or in moderate-strong alkaline protic solvents $(\mathrm{pH}=8 \sim 11)$ such as water or lower alcohols and in presence of a strong-mild oxidant (in most cases $\mathrm{H}_{2} \mathrm{O}_{2}$ ) and a suitable catalyst such as a metal ion or some kind of oxidoreductase enzyme, the excited 3-aminophthalate dianion (3-APA*) returns to the ground state (3-APA) by releasing energy in the form of light (Figure 1). ${ }^{17}$

In this study, a new chemiluminescence immunochromatographic analysis system with high sensitivity, and high reproducibility was developed for the determination of microcystins (MCs) in water. Horse radish peroxidase (HRP) labeled microcystin-LR monoclonal antibody (MCLR-mAb) was used for the sensitive chemiluminescence detection. The chemiluminescence immunochromatographic analysis system was composed

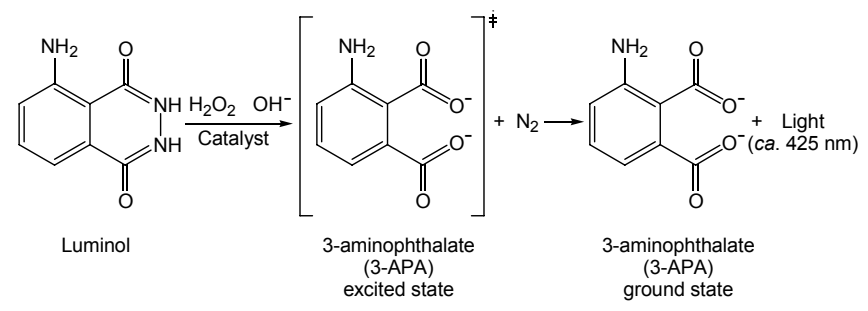

Figure 1. Structure of luminol (5-amino-2,3-dihydro-1,4-phthalazinedione) and luminol chemiluminescence reaction scheme. 
of MCLR-mAb-HRP conjugate, MCLR-BSA conjugate, luminol, hydrogen peroxide mixture solution, an immunochromatographic assay strip and luminometer.

\section{Experimental Section}

Chemicals. HRP (Horse Radish Peroxidase), luminol (5amino-2,3-dihydro-1,4-phthalazine-dione), hydrogen peroxide, sodium hydroxide, glutaraldehyde, BSA (Bovine Serum Albumin), Keyhole Limpet Hemocyanin (KLH), and 1-ethyl-3,3'dimethyl-aminopropyl carbodiimide (EDAC) were purchase from Sigma (St. Louis, MO). Proein G were purchased from Kem-En-Tec (Copenhagen, Denmark). Microcystin-LR monoclonal antibody (MCLR-mAb) and luminometer was supplied from Boditech Co. (Chunchon, South Korea). Microcystin-LR (MCLR) was extracted from microcystis aeruginosa in the Instrumental Anlytical Chemistry Laboratory, Kangwon National University (Chunchon, South Korea).

Production of Monoclonal Antibodies (mAb) against Microcystin-LR. To produce monoclonal antibodies $(\mathrm{mAb})$ against microcystin-LR, microcystin-LR was conjugated to bovine serum albumin (BSA) or keyhole limpet hemocyanin (KLH) in the presence of 1-ethyl-3,3'-dimethyl-aminopropyl-carbodiimide (EDAC). Hybridomas producing MCLR-mAb were prepared by a standard method ${ }^{8}$ for immunization and cell fusion Six-week-old mice were immunized with MCLR-KLH. The initial injection used $0.2 \mathrm{~mL}$ of the conjugate solution and 0.2 $\mathrm{mL}$ of complete Freund's adjuvant. Booster injections used conjugate solution and incomplete Freund's adjuvant. The mAb was produced in $\mathrm{BALB} / \mathrm{c}$ mice by the hybridoma cell line, $\mathrm{SP}_{2} /$ O-Ag14.

Two weeks after fusion, the hybridomas were screened for the production of anti-MCLR antibodies by an indirect ELISA in which the MCLR-protein conjugates were coated onto plates. Hybridomas were estimated as positive for the generation of specific antibodies in case they were positive for MCLR-BSA, MCLR-KLH. The positive hybridomas were cloned several

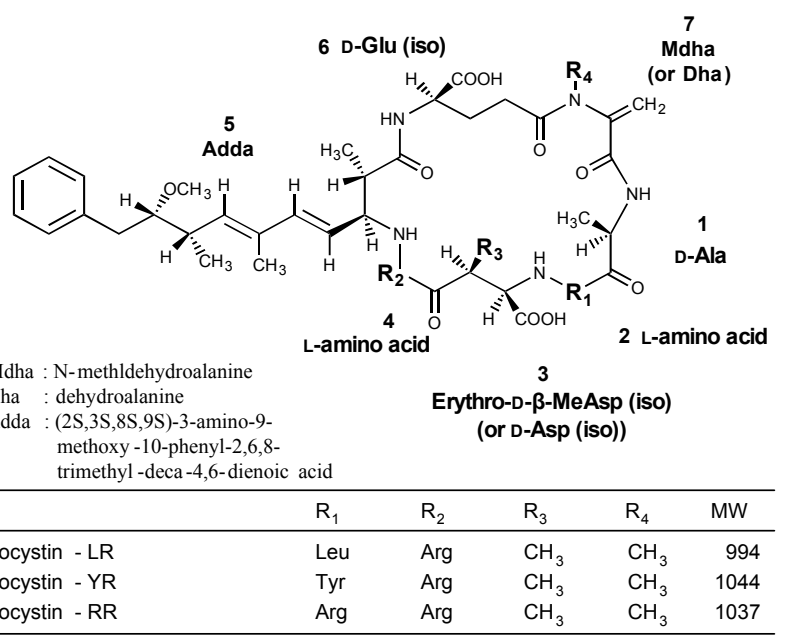

Figure 2. Structure of microcystins. A characteristic of microcystins and related cyanobacterial toxins is the hydrophobic amino acid Adda which contains in position 5 two conjugated double bonds. Numbers represent the positions of the corresponding amino acid. times by a limiting dilution method. Each of the established hybridoma cells producing the antibody was grown in medium supplemented with HT (Hydroxy Tryptamine). Large quantities of antibodies were prepared from serum-free cultured supernatants of hybridomas by membrane ultrafiltration, ammonium sulfate precipitation, and final purification using a protein $G$ column.

Preparation of MCLR-BSA Conjugation. $1 \mathrm{mg}$ BSA (Bovine Serum Albumin) was dissolved in $1 \mathrm{~mL}$ of $1 \mathrm{mM}$ PBS solution (pH 7.4), then $800 \mu \mathrm{L}$ of MCLR and $200 \mu \mathrm{L}$ of DMSO was added on the BSA solution. To conjugate MCLR and BSA, 11.5 $\mathrm{mg}$ of EDAC was added on the BSA solution, and the solution was incubated for 1 hour at room temperature. To remove unconjugated MCLR and BSA, the dialysis tubing was used in $250 \mathrm{~mL}$ PBS solution at room temperature for 2 hours. The dialysis process was repeated 4 times, and then supernatant was harvested by centrifugation at 13,000 rpm for 2 minutes.

Preparation of HRP Labeled MCLR-mAb. To prepare HRP labeled MCLR-mAb, MCLR-mAb and HRP were mixed into $1 \mathrm{~mL}$ of PBS solution ( $\mathrm{pH} 7.2$ ), then the mixed solution was stirred for 3 minutes at room temperature in the absence of light. Then $80 \mu \mathrm{L}$ of $1 \%$ glutaraldehyde solution was added on the mixed solution for the conjugation of MCLR-mAb and HRP. For the stopping the conjugation process, $108 \mu \mathrm{L}$ of $1 \mathrm{M}$ tris buffer solution ( $\mathrm{pH}$ 7.2) was used. To remove unconjugated HRP and MCLR-mAb, the dialysis tubing was used in $250 \mathrm{~mL}$ PBS solution at room temperature for 2 hours. The dialysis process was repeated 4 times, and then supernatant was harvested by centrifugation at 13,000 rpm for 2 minutes.

Chemiluminescence Immunoassay. The chemiluminescence immunoassay was conducted on the nitrocellulose membrane. The chemiluminescence detection zone is located on the nitrocellulose membrane (Millipore HF 180), and the bottom side of membrane was coated with a plastic thin film. The detection spot were located $10 \mathrm{~mm}$ left from the right end of the oval window. (Figure 3) $1 \mu \mathrm{L}$ of various concentrations of MCLRBSA conjugates was dispensed on the detection spot of the mem-

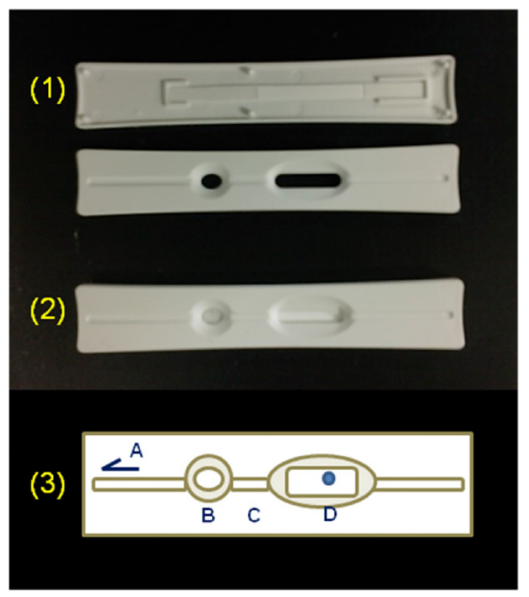

Figure 3. Chemiluminescence immunoassay strip. (1) Plastic housing $(15 \times 90 \mathrm{~mm})$ designed to fit to the holder of CL Scanner. (2) Assembled strip for chemiluminescence immunoassay. (3) Schematic diagram of chemiluminescence immunoassay strip. A: direction of insertion of the strip into CL scanner. B: absorption zone. C: lateral flow zone. D: detection zone. 


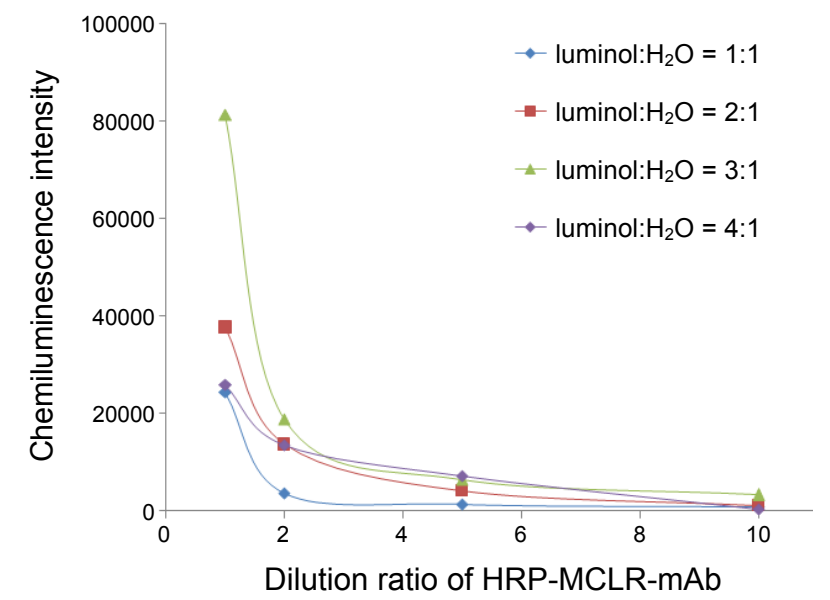

Figure 4. Titration curves of HRP-MCLR-mAb conjugate as a function of the ratio of luminol and hydrogen peroxide.

brane and the membrane was vacuum dried at $50{ }^{\circ} \mathrm{C}$ for $40 \mathrm{~min}$. Then, on the same spot, $1 \mu \mathrm{L}$ of HRP labeled MCLR-mAb was dispensed again. The membrane was vacuum dried again at $50{ }^{\circ} \mathrm{C}$ for $40 \mathrm{~min}$. After drying, the assembled strip onto a polystyrene card was placed into a plastic housing $(15 \times 90 \mathrm{~mm})$, which was designed to fit to the holder of CL Scanner. The width of oval window of plastic housing for the scanning of detection zone was $15 \mathrm{~mm}$. On the detection spot, $1 \mu \mathrm{L}$ of $2.6 \mathrm{mM}$ luminol- $\mathrm{H}_{2} \mathrm{O}_{2}$ solution was added and the emitted photons were measured.

To test the performance of chemiluminescence reaction in terms of the linear responses of luminol solution and different concentrations of HRP-MCLR-mAb, $1 \mu \mathrm{L}$ of diluted HRPMCLR-mAb conjugate was applied on the detection spot on the nitrocellulose membrane. Then the membrane was vacuum dried at $50{ }^{\circ} \mathrm{C}$ in a thermostat for $40 \mathrm{~min}$. After drying, $1 \mu \mathrm{L}$ of luminol solution was added and the emitted photons were measured for 1 minute. The area value of the CL peak at the detection zone was measured as CL intensity. For chemiluminescence measurement, $2.6 \mathrm{mM}$ luminol was dissolved in $0.1 \mathrm{M} \mathrm{NaOH}$, then $3 \% \mathrm{H}_{2} \mathrm{O}_{2}$ solution was added.

\section{Result and Discussion}

In order to clarify the relationship between the chemiluminescence intensity and concentration of HRP-MCLR-mAb conjugate, the concentration of luminol solution was kept constant and four different diluted HRP-MCLR-mAb solutions were used. Four different diluted HRP-MCLR-mAb solutions (1:1,
$1: 2,1: 5,1: 10)$ were prepared by diluting with $0.01 \mathrm{M}$ tris buffer (pH 6.5). 1:2 means the mixed solution of 1 volume of HRPMCLR-mAb solution and 2 volume of $0.01 \mathrm{M}$ tris buffer $(\mathrm{pH}$ 6.5). Therefore, $1: 1$ solution is 10 times more concentrated than 1:10 solution. Table 1 shows the relationship between chemiluminescence intensity and concentration of HRP-MCLR-mAb. 1:1 solution showed the largest chemiluminescence intensity while 1:10 solution showed the smallest. Increasing the HRPMCLR-mAb concentration resulted in the increased chemiluminescence intensity.

For improvement of the sensitivity in chemiluminescence detection, it is essential to find the optimum ratio of luminol and $\mathrm{H}_{2} \mathrm{O}_{2}$. To find the optimum ratio of luminol and four different ratios of luminol solutions (luminol: $\mathrm{H}_{2} \mathrm{O}_{2}=1: 1,2: 1,3: 1$, $4: 1)$ were tried. In this experiment, the same four different diluted HRP-MCLR-mAb solutions $(1: 1,1: 2,1: 5,1: 10)$ as the previous experiment were used for each luminol/hydrogen peroxide ratio. An aliquot of $1 \mu \mathrm{L}$ MCLR-BSA conjugate $(1.1 \mathrm{ng} /$ $\mathrm{mL}$ ) was spoted on the detection spot of the nitrocellulose membrane, and a HRP-MCLR-mAb solution of a given concentration was applied on the same spot. The chemiluminescence produced on the detection spot was measured. Results were shown in Table 2. According to Table 2, 3:1 ratio seems to be

Table 2. Chemiluminescence intensity as a function of mixed ratio of luminol: $\mathrm{H}_{2} \mathrm{O}_{2}$ and dilution ratio of HRP-MCLR-mAb

\begin{tabular}{ccc}
\hline $\begin{array}{c}\text { Dilution ratio } \\
\text { of HRP-MCLR-mAb }\end{array}$ & $\begin{array}{c}\text { Ratio of } \\
\text { Luminol: } \mathrm{H}_{2} \mathrm{O}_{2}\end{array}$ & $\begin{array}{c}\text { Chemiluminescence } \\
\text { intensity }\end{array}$ \\
\hline $1: 1$ & $1: 1$ & $2.441 \times 10^{4}$ \\
& $2: 1$ & $3.763 \times 10^{4}$ \\
$3: 1$ & $8.128 \times 10^{4}$ \\
& $4: 1$ & $2.579 \times 10^{4}$ \\
$1: 2$ & $1: 1$ & $3.519 \times 10^{4}$ \\
& $2: 1$ & $1.360 \times 10^{4}$ \\
& $3: 1$ & $1.883 \times 10^{4}$ \\
& $4: 1$ & $1.353 \times 10^{4}$ \\
& $1: 1$ & $1.280 \times 10^{3}$ \\
& $2: 1$ & $4.092 \times 10^{3}$ \\
& $3: 1$ & $6.347 \times 10^{3}$ \\
& $4: 1$ & $7.124 \times 10^{3}$ \\
& $1: 1$ & $8.257 \times 10^{2}$ \\
& $2: 1$ & $1.129 \times 10^{2}$ \\
& $3: 1$ & $3.158 \times 10^{2}$ \\
& $4: 1$ & $2.543 \times 10^{2}$ \\
\hline
\end{tabular}

${ }^{a}$ Dilution solution for HRP-MCLR-mAb was 0.01 M Tris buffer (pH 6.5).

Table 1. Chemiluminescence intensity as a function of dilution ratio of HRP-MCLR-mAb

\begin{tabular}{|c|c|c|c|c|c|c|}
\hline \multirow{2}{*}{$\begin{array}{c}\text { Dilution of } \\
\text { HRP-MCLR-mAb }\end{array}$} & \multicolumn{3}{|c|}{ Chemiluminescence intensity } & \multirow[b]{2}{*}{ Average } & \multirow[b]{2}{*}{$\mathrm{SD}$} & \multirow[b]{2}{*}{$\operatorname{RSD}(\%)$} \\
\hline & 1 & 2 & 3 & & & \\
\hline $1: 1$ & $1.026 \times 10^{4}$ & $1.080 \times 10^{4}$ & $1.075 \times 10^{4}$ & $10.6 \times 10^{3}$ & $0.3 \times 10^{3}$ & 3 \\
\hline $1: 2$ & $8.161 \times 10^{3}$ & $8.581 \times 10^{3}$ & $7.828 \times 10^{3}$ & $8.2 \times 10^{3}$ & $0.4 \times 10^{3}$ & 5 \\
\hline $1: 5$ & $2.380 \times 10^{3}$ & $2.634 \times 10^{3}$ & $2.724 \times 10^{3}$ & $2.6 \times 10^{3}$ & $0.2 \times 10^{3}$ & 7 \\
\hline $1: 10$ & $1.040 \times 10^{2}$ & $1.000 \times 10^{2}$ & $1.110 \times 10^{2}$ & $10.5 \times 10$ & $0.6 \times 10$ & 5 \\
\hline
\end{tabular}

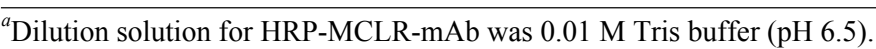


Table 3. Chemiluminescence intensity against different concentrations of MCLR - BSA

\begin{tabular}{|c|c|c|c|c|c|c|}
\hline \multirow{2}{*}{$\begin{array}{c}\text { Concentration of } \\
\text { MCLR-BSA }(\mathrm{pg} / \mathrm{mL})\end{array}$} & \multicolumn{3}{|c|}{ Chemiluminescence intensity } & \multirow{2}{*}{ Average } & \multirow{2}{*}{ SD } & \multirow{2}{*}{$\begin{array}{l}\text { RSD } \\
(\%)\end{array}$} \\
\hline & 1 & 2 & 3 & & & \\
\hline 4.73 & $7.680 \times 10^{2}$ & $6.130 \times 10^{2}$ & $7.840 \times 10^{2}$ & $7.2 \times 10^{2}$ & $0.9 \times 10^{2}$ & 13 \\
\hline 9.45 & $9.280 \times 10^{2}$ & $9.140 \times 10^{2}$ & $8.050 \times 10^{2}$ & $8.8 \times 10^{2}$ & $0.7 \times 10^{2}$ & 8 \\
\hline 47.3 & $1.180 \times 10^{3}$ & $1.181 \times 10^{3}$ & $1.213 \times 10^{3}$ & $11.9 \times 10^{2}$ & $0.2 \times 10^{2}$ & 2 \\
\hline 94.5 & $1.406 \times 10^{3}$ & $1.387 \times 10^{3}$ & $1.439 \times 10^{3}$ & $14.1 \times 10^{2}$ & $0.3 \times 10^{2}$ & 2 \\
\hline 945 & $3.030 \times 10^{3}$ & $3.025 \times 10^{3}$ & $3.168 \times 10^{3}$ & $30.7 \times 10^{2}$ & $0.8 \times 10^{2}$ & 3 \\
\hline
\end{tabular}

$\mathrm{NC}$ membrane were spotted with $1 \mu \mathrm{L}$ of ten times dilution of the HRP-MCLR-mAb using $0.01 \mathrm{M}$ Tris buffer (pH 6.5), using mixed ratio of Luminol: $\mathrm{H}_{2} \mathrm{O}_{2}=3: 1$.

the optimum luminol/hydrogen peroxide ratio for the sensitive chemiluminescence detection. From 1:1 to 3:1 ratio, we could observe that higher luminol ratio resulted in an increase in the chemiluminescence intensity. However, in the case of 4:1 ratio, a severe decrease in the chemiluminescence intensitiy was observed because of too low composition of hydrogen peroxide. The role of hydrogen peroxide is to produce an oxidized HRP. The oxidized HRP can return the excited state (3-APA*) to the ground state (3-APA) of luminol, resulting in releasing energy in the form of light. Consequently, decrease of the oxidized HRP compound owing to retarded oxidation should cause the decrease in chemiluminescence intensitiy.

Finally, by using chemiluminescence immunochromatography, we analyzed the concentration of MCLR-BSA conjugate in tris buffer solution. MCLR-BSA conjugate samples are prepared as follows: MCLR-BSA conjugate was diluted with $0.01 \mathrm{M}$ tris buffer ( $\mathrm{pH}$ 6.5) to produce five different concentrations ( 945 $\mathrm{pg} / \mathrm{mL}, 94.5 \mathrm{pg} / \mathrm{mL}, 47.25 \mathrm{pg} / \mathrm{mL}, 9.45 \mathrm{pg} / \mathrm{mL}, 4.73 \mathrm{pg} / \mathrm{mL}$ ) of samples. The results were summarized in Table 3 . Since from Table 3 , in the case of the concentration of $4.73 \mathrm{pg} / \mathrm{mL}, \mathrm{RSD}$ value was much higher than the other concentrations, we could conclude that with this chemiluminescence assay, MCLR can be reproducible detected in water at a concentration as low as $9.45 \mathrm{pg} / \mathrm{mL}$. The chemiluminescence assay method would have a great advantage in terms of low detection limit compared to other analytical methods. For the quantitative analysis of MCLR in water sample, HPLC and ELISA (Enzyme Linked ImmunoSorbent Assay) are the most popular analytical tools today. However, using such analytical tools, it is impossible to obtain a quantitative analysis result with a low concentration of MCLR sample since the detection limits of HPLC and ELISA are 420 $\mathrm{pg} / \mathrm{mL}^{18}$ and $250 \mathrm{pg} / \mathrm{mL}^{19}$ individually.

The aim of this study was an application of the chemiluminescence immunochromatography on the detection of microcystins in water samples. The detection sensitivity of the chemiluminescence immunochromatography using monoclonal antibodies was high enough to utilize for the measurement of microcystin content in water. Since the chemiluminescence immunochromatography assay is easy to perform and their quantitative range is large, it shows a potential for a powerful tool in quantitative analysis of microcystins in water samples.

Acknowledgments. This work was supported by the EcoSTAR project grant funded by the Korea government (Ministry of Environment).

\section{References}

1. Thorpe, G. H. G.; Kricka, L. J. In Biolumin. Chemilumin.: New Perspectives; Scholmerich, J. R., Andreesen, R., Kapp, A., Ernst, M., Woods, W., eds.; Chichester: J. Wiley. P. 1987; pp 199-208.

2. Paradies, H. H.; Gesellschaft, B. D. B. Phys. Chem. 1992, 96, 1027.

3. Zhang, X. R.; Baeyens, W. R. G.; Garcia-Campana, A. M.; Quyang, J. Trends Anal. Chem. 1999, 18, 6.

4. Maccapra, F. Oxford University Press: Oxford, 1984; pp 617-637.

5. Pyo, D.; Huang, Y.; Kim, Y.; Hahn, J. H. Bull. Korean Chem. Soc. 2005, 26, 939.

6. Oh, H. M.; Lee, S. J.; Kim, J. H.; Yoon, B. D. Appl. Envir. Micro. 2002, 67, 1484.

7. Pyo, D.; Yoo, J. J. Immun. Immunochem. 2008, 29, 211.

8. Ha, S. G.; Park, J. B.; Ko, K. K.; Choi, E. Y. Hybridoma. 1999, 18 , 371.

9. Pyo, D.; Moon D. Bull. Korean Chem. Soc. 2005, 26, 2089.

10. Pyo, D.; Yoo, J. Anal. Lett. 2007, 40, 3208.

11. Egorov, A. M.; Kim, B. B.; Pisarev, V. V.; Kapeliuch, Yu. L.; Gazarian, I. G. In Biolum and Chemilum. Status report; Szalay, A. A., Stanly, P. E., Kricka, L. J., eds.; Chichester: Wiley. P. 1993; pp 286-290.

12. Yamaguchi, M.; Yoshida, H.; Nohta, H. J. Chromatogr. A 2002, 950, 1.

13. White, E. H., McElroy, W. D., Glass, B., Eds.; Light and Life, John Hopkins Press: Baltimore, 1961; pp 183-195.

14. White, E. H.; Bursey, M. M. J. Am. Chem. Soc. 1964, 86, 941.

15. White, E. H.; Zafiriou, O.; Kagi, H. H.; Hill, J. H. M. J. Am. Chem. Soc. 1964, 86, 940.

16. Gundermann, K. D.; McCapra, F. Chemiluminescence in Organic Chemistry; Springer-Verlag: Berlin, 1987.

17. Barni, F.; Lewis, S. W.; Berti, A.; Miskelly, G. M.; Lago, G.; Talanta 2007, 72, 896.

18. Congying, G.; Li, L.; Nenghu, F.; Jinping, J. Chin. J. Chromatogr. 2007, 25, 174

19. Bouarcha, N.; Maatouk, I.; Vincent, G.; Levi, Y. Toxicol. 2002, 40, 1677. 